Advances in Applied Physics, Vol. 1, 2013, no. 1, 29 - 38

HIKARI Ltd, www.m-hikari.com

\title{
Logic Gates Based on Soliton Transmission in the Toda Lattice
}

\author{
Yoji Kubota \\ Department of Chemistry, Kyushu University \\ Fukuoka 812-8581, Japan \\ ykubota@chem.kyushu-univ.jp \\ Takashi Odagaki \\ Department of Physics, Tokyo Denki University \\ Saitama 350-0394, Japan
}

Copyright (C) 2013 Yoji Kubota and Takashi Odagaki. This is an open access article distributed under the Creative Commons Attribution License, which permits unrestricted use, distribution, and reproduction in any medium, provided the original work is properly cited.

\begin{abstract}
We present conceptual models for fundamental logic gates using solitons as bit pulses in the Toda lattice. The transmission rate of the soliton is controllable by another soliton and the control of the soliton transmission is applicable to a NOT gate. Two-input system with appropriate transmission filters were constructed to realize AND and OR gates. We numerically demonstrated the soliton transmissions on these systems. The efficiency of our logic gates was improved for larger amplitude of the input solitons.
\end{abstract}

Keywords: soliton, logic gate, resonant transmission

\section{Introduction}

One of the most important application of soliton transmission is soliton communication in optical fibers $[1,8]$. In the optical communication, the soliton is used as a bit of information and states " 1 " and " 0 " are assigned to the presence and absence of the soliton, respectively. When the soliton bit is controllable 


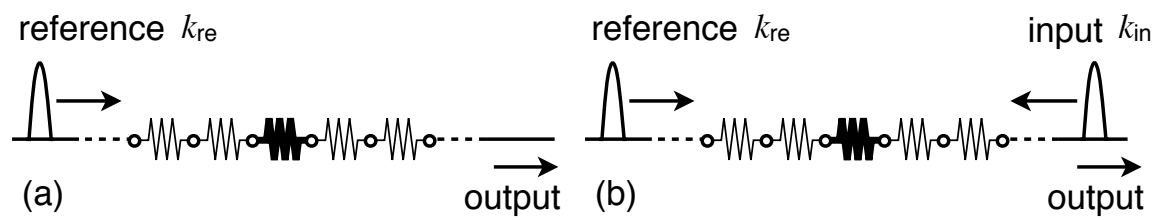

Figure 1: Schematic diagram of incident waves on two identical Toda lattices connected by a harmonic spring for (a) the 0-input and (b) 1-input initial conditions. The thin springs are the Toda lattices and the thick spring is a harmonic spring. We observed a transmitted right-moving wave on the right side of the system as an output signal.

by another soliton, logic gates can be constructed, and all-soliton communication and computing system would be realized. Several authors suggested soliton logic gates using the interference effects between the solitons traveling together $[2,13]$ and the phase shift due to the soliton-soliton scattering $[4,12]$.

In this paper, we present conceptual models for logic gates based on the control of the soliton transmission rate in the Toda lattice. The Toda lattice is a well-known integrable system, which supports exact soliton solutions [14]. The transmission and scattering of the soliton in the Toda lattice with a local defect were studied by many authors theoretically [17], numerically [11, 10], and using a $L C$ ladder circuit [16]. Recently, it was proposed to enhance the soliton transmission rate by controlling the defect appropriately $[6,7,15]$. We consider the control of the transmission rate by using another soliton based on the resonant transmission of the soliton [6] to construct fundamental logic gates. A NOT gate is realized by using the control-bit soliton to interfere the resonant transmission of the soliton. The resonant soliton transmission is also applicable to soliton filters of amplitude transmittance for AND and OR gates. We numerically demonstrated these logic gates work properly and showed the efficiency of the logic gates by comparing the amplitude of the output solitons.

\section{Model}

To construct a NOT gate, we considered two identical Toda lattices [14] connected by a harmonic spring. The equation of motion is given by

$$
m \frac{\mathrm{d}^{2} q_{n}}{\mathrm{~d} t^{2}}=-f_{n}+f_{n-1},
$$


where $m$ and $q_{n}$ are the mass and displacement of the $n$-th particle, respectively. The interaction forces $f_{n}$ are

$$
f_{n}= \begin{cases}a\left[\exp \left(-b r_{n}\right)-1\right] & \text { for } n \neq 0 \\ -\kappa r_{n} & \text { for } n=0\end{cases}
$$

where $r_{n}\left(\equiv q_{n+1}-q_{n}\right)$ is a relative displacement, $a$ and $b$ are constants of the Toda spring, and $\kappa$ is a spring constant of the harmonic spring. Since we used the identical homogeneous Toda lattices, it was possible to set $m=a=$ $b=1$ using dimensionless variables defined in our previous papers $[5,6]$. We considered two initial conditions denoted by "0-input" and "1-input". For the 0 -input, we prepared a right-moving single soliton at time $t=0$ as a reference pulse (see Fig. 1(a)). The reference soliton was given by

$$
\begin{aligned}
f_{n} & =\frac{\sinh ^{2} k}{\cosh ^{2}\left[k\left(n-n_{0}\right)-\omega t\right]}, \\
\omega & =\sinh k,
\end{aligned}
$$

with a wave number $k=k_{\text {re }}$ and an initial location $n_{0}$ set so that the reference soliton was far left from the harmonic spring. The spring constant of the harmonic spring was chosen to be satisfied with the resonant condition [6] for the reference soliton. We denote the spring constant in the resonant condition by $\kappa_{\text {reso }}\left(k_{\text {re }}\right)$. As a result of the resonant condition, the reference soliton passes almost completely the harmonic spring without much decay. After a sufficient time, we observe the transmitted right-moving soliton on the right side of the system as an output signal. For the 1 -input, at time $t=0$, we prepared two solitons as reference and input-signal pulses on either hand of the harmonic spring (see Fig. 1(b)). The reference soliton was identical to that for the 0input. The input-signal soliton was a left-moving soliton given by Eq. (4) with $k=k_{\text {in }}, n_{0} \gg 1$, and $\omega=-\sinh k_{\text {in }}$. The specific value of $n_{0}$ for the inputsignal soliton was set to its optimal value, which we searched numerically for the efficiency of the logic gate. The input-signal soliton disturbs the resonant condition between the reference soliton and the harmonic spring. As a result, the output-signal soliton is smaller than that for the 0-input. Therefore, this system would work as a NOT gate by controlling the transmission of the reference soliton with or without the input-signal soliton. To check the efficiency of the NOT gate, we calculated the amplitude of the output-signal soliton $F_{\text {out }}^{(*)}$, where the superscript indicates the initial condition. However, it seems as if the soliton amplitude fluctuates with the propagation because the peak of the traveling soliton does not necessarily coincide with a lattice site. To avoid this artificial fluctuation, we observed the maximal value of $f_{n}(t)$ as a function of time at a fixed site chosen appropriately to obtain $F_{\text {out }}^{(*)}$. the amplitude of the output-signal soliton. 

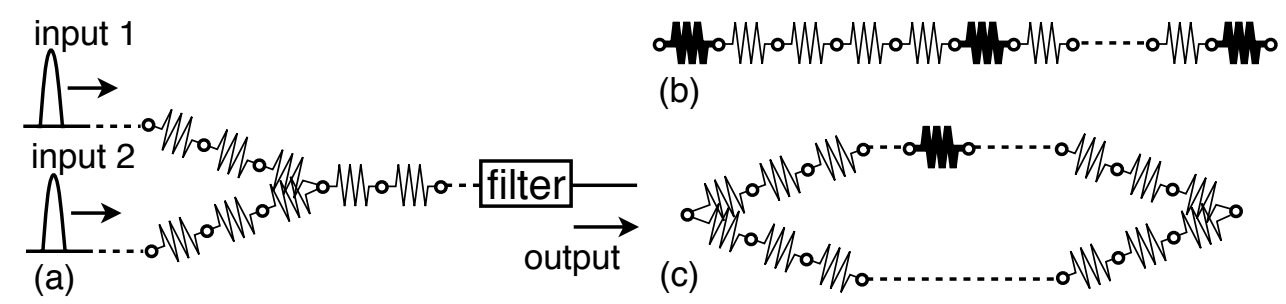

(b)

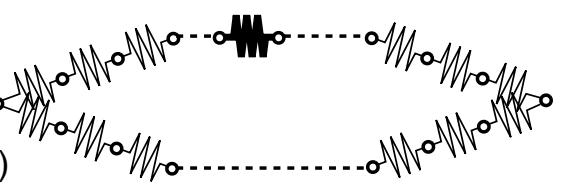

Figure 2: (a) Schematic diagram of a two-input system consisting of the Toda lattices with a filter. (b) The AND-gate filter consists the Toda lattice, where the Toda springs at every five sites are replaced by the harmonic springs having $\kappa_{\text {reso }}\left(k_{\text {in }}\right)$. (c) The OR-gate filter consists of the branched Toda lattice. In the upper branch, one of the Toda spring is replaced by the harmonic spring having $\kappa_{\text {reso }}\left(k_{10}\right) / 2$.

We also considered other model to construct AND and OR gates. Two identical Toda lattices were used as two input lines and converged at $n=0$ into one Toda lattice, which was used as an output line with a transmission filter (see Fig. 2(a)). We set $m=a=1 / 2$ and $b=1$ in the Toda lattice of the branched region (for $n \leq-1$ ) and the parameters in the other region were set to be $m=a=b=1$. The equation of motion for the joint (at $n=0$ ) is given by

$$
\frac{\mathrm{d}^{2} q_{n}}{\mathrm{~d} t^{2}}=-\exp \left(-r_{n}\right)+\frac{1}{2} \exp \left(-r_{n-1}^{(\mathrm{u})}\right)+\frac{1}{2} \exp \left(-r_{n-1}^{(\mathrm{l})}\right)
$$

where $r^{(\mathrm{u})}$ and $r^{(\mathrm{l})}$ denotes the relative displacements for the upper and lower branches, respectively. We considered two initial conditions: (i) two identical solitons with $k_{\text {in }}$ prepared on the both input lines and (ii) a single soliton with $k_{\text {in }}$ prepared only on the upper branch. We denote the former and latter initial conditions by "11-input" and "10-input", respectively. For the 11-input, the motions of the upper and lower branches are synchronized with each other. In this case, Eq. (5) is strictly reduced to the equation of motion for the homogeneous Toda lattice, and therefore, the incident solitons for the 11-input completely pass the joint. On the other hand, the incident soliton for the 10input is scattered by the joint and the wave number of the transmitted soliton becomes smaller (denoted by $k_{10}$ ).

The two-input system works as AND and OR gates by using appropriate transmission filters, as we describe below. The basic idea for the AND-gate filter is using the harmonic spring with $\kappa_{\text {reso }}\left(k_{\text {in }}\right)$, which the transmitted soliton of the 11-input passes without much decay. For a more efficient AND-gate filter, we embedded 21 harmonic springs with $\kappa_{\text {reso }}\left(k_{\text {in }}\right)$ on every five sites of the Toda lattice (see Fig. $2(\mathrm{~b})$ ). The soliton having $k_{\text {in }}$ passes the filter, whereas 

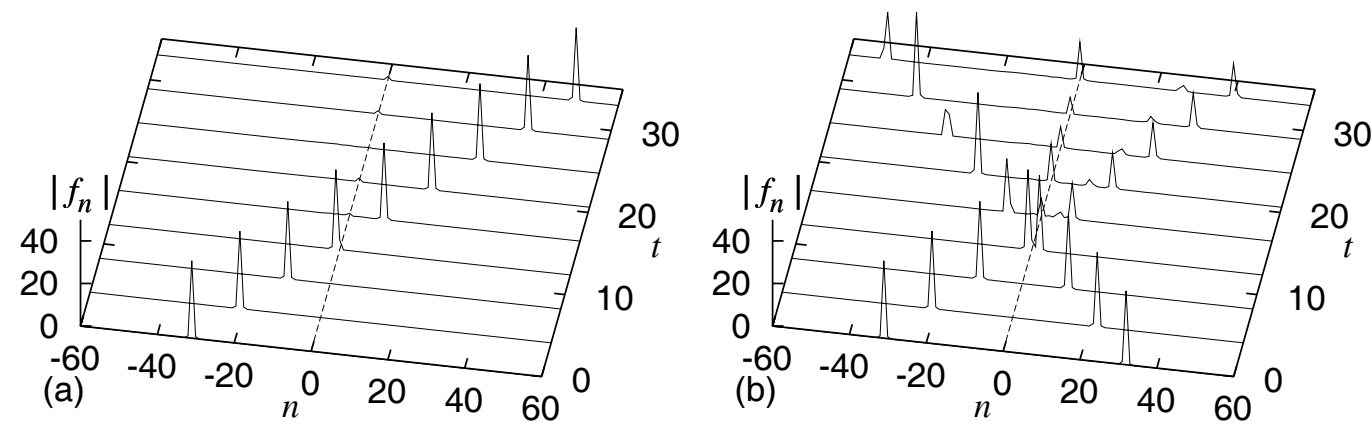

Figure 3: Time evolution of the solitons for (a) the 0-input and (b) 1-input initial conditions. We set $k_{\mathrm{re}}=2.5$ and $n_{0}=-31$ for the reference soliton, and $k_{\text {in }}=2.5$ and $n_{0}=31.9139$ for the input-signal soliton. The dashed line at $n=0$ denotes the location of the harmonic spring with $\kappa_{\text {reso }}\left(k_{\text {re }}\right)=7.776$.

the smaller soliton having $k_{10}$ diminishes gradually at every harmonic springs. On the other hand, the harmonic spring with $\kappa_{\text {reso }}\left(k_{10}\right)$ is applicable to the OR-gate filter. For the OR gate, we used a branched line made of the Toda lattices with $m=a=1 / 2$, where one of the Toda spring on the upper branch was replaced by the harmonic spring with $\kappa=\kappa_{\text {reso }}\left(k_{10}\right) / 2$ (see Fig. $2(\mathrm{c})$ ). The coefficient $1 / 2$ was added to match with $a=1 / 2$. The soliton having $k_{10}$ passes the filter without much decay, whereas that having $k \neq k_{10}$ is scattered by the filter. As a result, a large soliton reduces its own wave number and the branched line could work as the OR-gate filter.

\section{$3 \quad$ Numerical Results}

We integrated Eq. (1) for the NOT gate system numerically using a third-order bilateral symplectic algorithm [3]. We set integration step $\Delta t \leq 0.01$ so that total energy did not deviate from the value of the initial state. In Fig 3, we show the spacetime evolution of $\left|f_{n}\right|$ for (a) the 0-input and (b) 1-input with $k_{\mathrm{re}}=k_{\text {in }}=2.5$. Figure 3(a) shows that the reference soliton prepared at $n=$ -31 moved to the right and reached the harmonic spring at $t \simeq 12$. Since $\kappa$ was set to be satisfied with the condition of the resonant transmission, the reference soliton passed the harmonic spring almost completely. In Fig. 3(b), the rightmoving reference soliton and the left-moving input-signal soliton traveled to the harmonic spring from each side of the system and collided with each other near the harmonic spring. As described above, the initial location $n_{0}$ of the input-signal soliton was chosen to minimize $F_{\text {out }}^{(1)}$ and, as a result, $F_{\text {out }}^{(1)}$ decreased almost by half of that for the 0-input.

To discuss efficiency of the NOT gate, we calculated the ratio $\alpha$ of the 

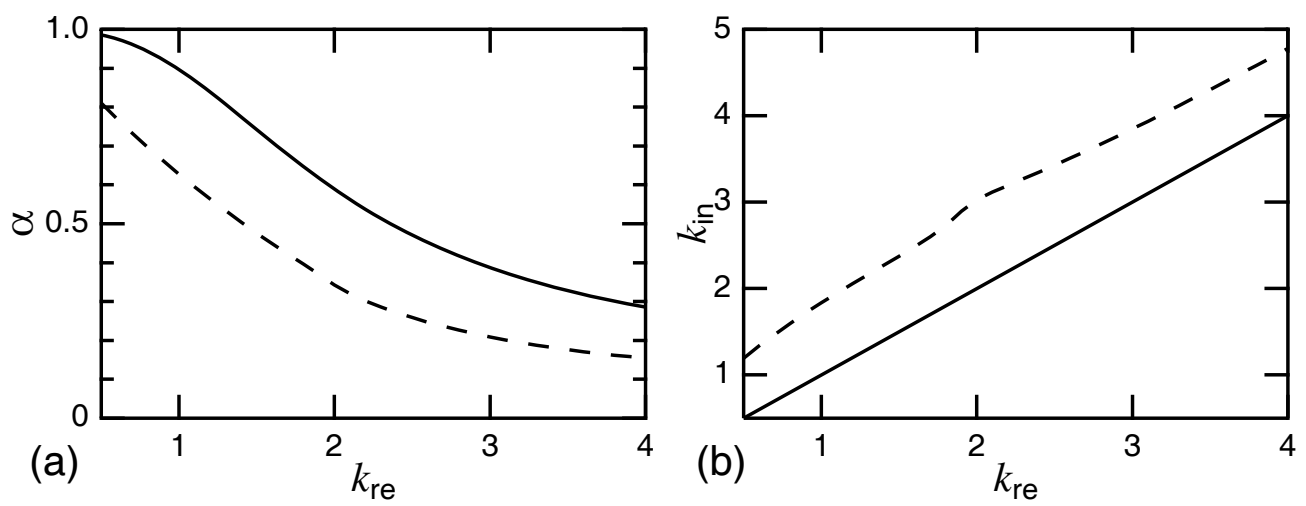

Figure 4: (a) The ratio $\alpha$ of the amplitude of the output-signal soliton for the 0 -input and 1-input as a function of $k_{\mathrm{re}}$. The solid and dashed curves are $\alpha$ for $k_{\text {in }}=k_{\text {re }}$ and for the optimized $k_{\text {in }}$, respectively. (b) The optimal values of $k_{\text {in }}$ are plotted by the dashed curve. The solid line is $k_{\text {in }}=k_{\text {re }}$ and is drawn for reference.

amplitude of the output-signal solitons for the two different initial conditions, i.e., $\alpha=F_{\text {out }}^{(1)} / F_{\text {out }}^{(0)}$. The solid curve in Fig. 4(a) shows the $k_{\text {re }}$-dependence of $\alpha$ for $k_{\text {in }}=k_{\text {re }} . \alpha$ decreased with increasing $k_{\text {re }}$, and therefore larger soliton suits for the signal bit of the NOT gate. To obtain a more efficient NOT gate, we optimized $k_{\text {in }}$ to minimize the output-signal soliton for the 1-input. As shown in Fig. $4(\mathrm{~b})$, the optimal $k_{\text {in }}$ were determined to be finite values and therefore the efficiency of the NOT gate was improved (see the dashed curve in Fig. 4(a)).

On the two-input system, we calculated the time evolution of the waves. Without the transmission filters, the incident soliton for the 11-input completely passed the joint (at $n=0$ ) and the soliton traveled in the output line as a single soliton keeping the wave number $k_{\text {in }}$ unchanged. For the 10input, the incident soliton was scattered at the joint and was divided into the transmitted soliton, a reflected soliton, and many small waves. As shown in Fig. $5, k_{10}$ was smaller than the wave number of the transmitted soliton for the 11-input given by $k_{\text {in }}$.

By using the transmission filters, we modulated the transmitted solitons to construct AND and OR gates. We embedded the AND-gate filter in $n=$ $[10,100]$ and calculated the wave propagation. Figure $6(\mathrm{a})$ shows that the transmitted soliton of the 11-input passed each harmonic spring without much decay since $\kappa$ were set to resonate with the soliton having $k_{\text {in }}$. On the other hand, the transmitted soliton of the 10-input was scattered at each harmonic spring and decreased its amplitude gradually (see Fig. 6(b)). Thus, the outputsignal soliton for the 10-input was significantly smaller than that for the 11- 


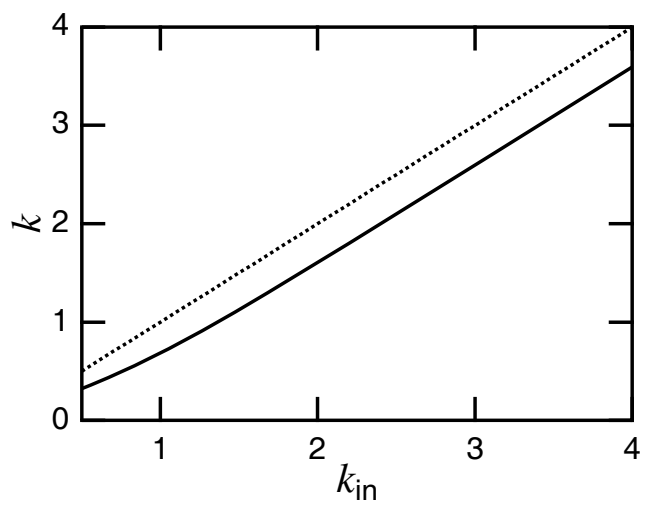

Figure 5: The wave number of the transmitted soliton in the two-input system without the transmission filters for the 10-input (solid) and for the 11-input (dotted). The former is denoted by $k_{10}$ in the text and the latter is given by $k=k_{\text {in }}$.

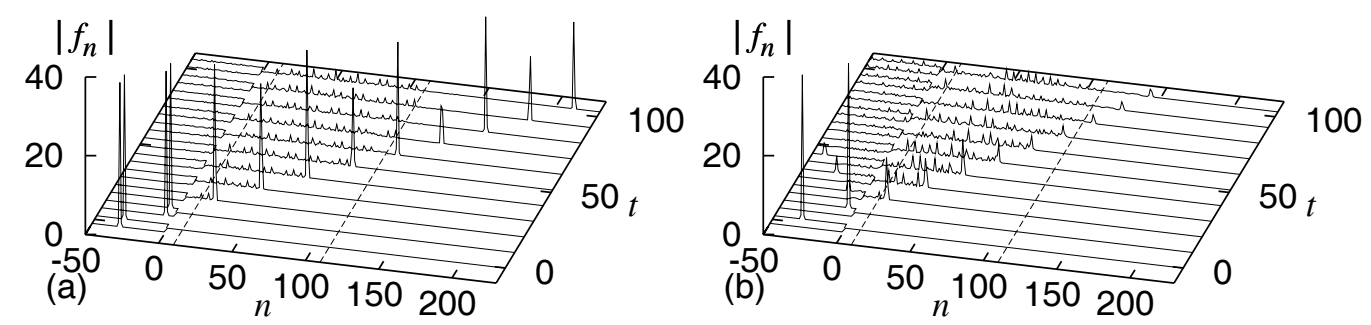

Figure 6: The spacetime evolution of $\left|f_{n}\right|$ in the two-input system with the AND-gate filter located at $n=[10,110]$ for (a) the 11-input and (b) 10-input initial conditions with $k_{\text {in }}=2.5$ and $n_{0}=-31$. For clarity, in the branched region, $\left|f_{n}\right|$ is scaled by $a=1 / 2$, and the waves on each line are shown with deviating slightly in the time coordinate. The region of the AND-gate filter is indicated by the dashed lines.

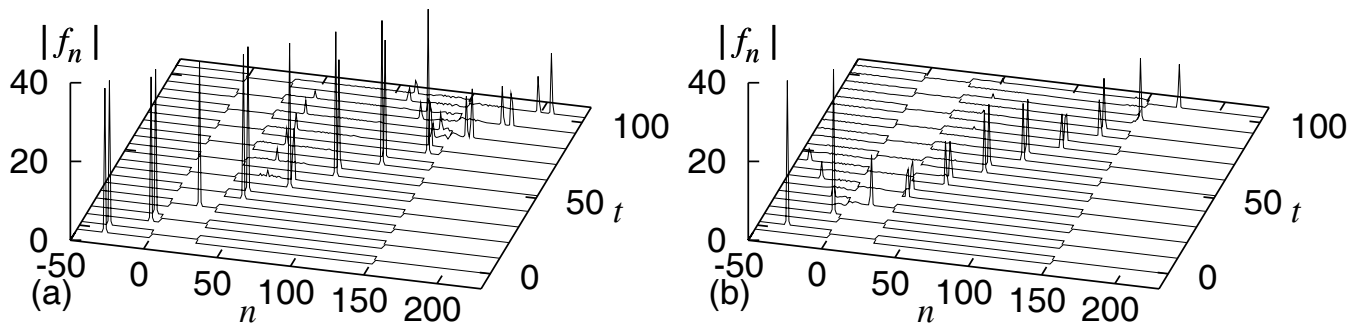

Figure 7: The same as Fig. 6 but with the OR-gate filter located at $n=$ $[30,152]$. 


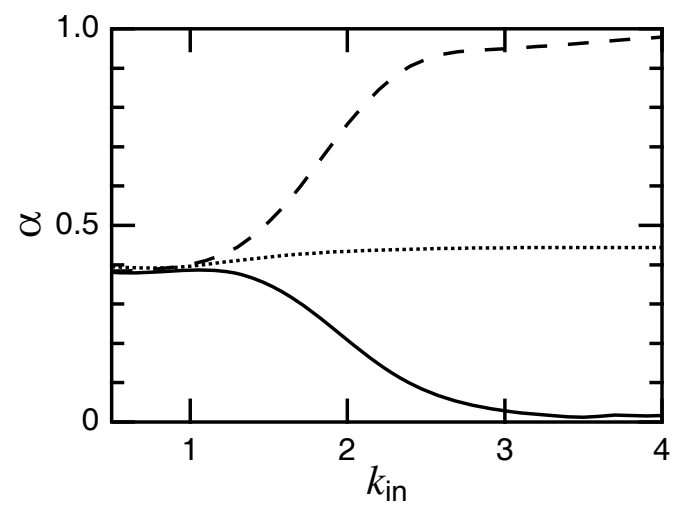

Figure 8: $k_{\text {in }}$-dependence of $\alpha\left(=F_{\text {out }}^{(10)} / F_{\text {out }}^{(11)}\right)$ without the filter (dotted curve), with the AND-gate filter (solid curve), and with the OR-gate filter (dashed curve).

input and this system would work as a AND gate. For the OR gate, we prepared the OR-gate filter at $n=[30,152]$, where the Toda spring at $n=51$ on the upper branch was replaced by the harmonic spring with $\kappa_{\text {reso }}\left(k_{\text {re }}\right) / 2$. Figure 7(b) shows that the initial soliton of the 10-input was scattered by the first joint (at $n=0$ ) and changed its wave number into $k_{10}$. In the next branched region (the OR-gate filter), the solitons having $k_{10}$ traveled each path and the soliton in the upper branch resonantly passed the harmonic spring. Then, the incident soliton left the OR-gate filter without incident and the wave number of the output-signal soliton was unchanged, i.e., $k \simeq k_{10}$. For the 11-input (see Fig. 7(a)), the incident solitons entered the OR-gate filter with keeping its wave number $k_{\text {in }}$ unchanged. In the upper branch path, the soliton was scattered by the harmonic spring and decelerated, and then, it reached the exit of the filter a bit later than that traveled in the lower branch path. After that, by the scattering at the exit of the filter, the wave number of the faster soliton was reduced to $k \simeq k_{10}$. Therefore, the OR-gate filter changes the wave number of the transmitted soliton into $k_{10}$ for both initial conditions, i.e., $F_{\text {out }}^{(10)} \simeq F_{\text {out }}^{(11)}$.

In Fig. 8, we show $\alpha$ given by $F_{\text {out }}^{(10)} / F_{\text {out }}^{(11)}$ for various $k_{\text {in }}$ to see the efficiency of the AND- and OR-gate filters. The solid curve in Fig. 8 denotes $\alpha$ obtained without the filters and is plotted for reference. This figure shows that for small $k_{\text {in }}$, the transmission filters hardly affected the transmitted soliton. $\alpha$ deviated from each other with increasing $k_{\text {in }}$ for $k_{\text {in }} \gtrsim 1$. Around $k_{\text {in }} \simeq 2.5, \alpha$ for the AND- and OR-gate filters were close to 0 and 1, respectively. Thus, the filters worked well for larger $k_{\text {in }}$ and the efficiencies were almost saturated to the best values for $k_{\text {in }} \gtrsim 2.5$. 


\section{Concluding Remarks}

We have presented a conceptual models to realize the fundamental logic gates using the soliton as the bit pulses in the lattice system. Our numerical results showed the amplitude of the output signals were controlled properly into the desired values determined by the input signals and thus, our models worked as the logic gates. It is well known that the Toda lattice is realized by using the nonlinear $L C$ circuit, and the present works are tested by the $L C$ circuit $[9,6]$.

\section{References}

[1] G. P. Agrawal, Nonlinear Fiber Optics, Academic Press, San Diego, 3rd edition, (2001).

[2] G. Cancellieri, F. Chiaraluce, E. Gambi, and P. Pierleoni, Coupled-soliton photonic logic gates: practical design procedures, J. Opt. Soc. Am. B, 12 (1995), 1300-1306.

[3] L. Casetti, Efficient symplectic algorithms for numerical simulations of Hamiltonian flows, Phys. Scr., 51 (1995), 29-34.

[4] M. H. Jakubowski, K. Steiglitz, and R. Squier, State transformations of colliding optical solitons and possible application to computation in bulk media, Phys. Rev. E, 58 (1998), 6752-6758.

[5] Y. Kubota and T. Odagaki, Propagation of solitons in the Toda lattice with an impure segment, Phys. Rev. E, 61 (2000), 3133-3138.

[6] Y. Kubota and T. Odagaki, Resonant transmission of a soliton across an interface between two Toda lattices, Phys. Rev. E, 71 (2005), 016605.

[7] Y. Kubota and T. Odagaki, Delay in a soliton transmission across an interface between two Toda lattices, J. Phys. A: Math. Gen., 39 (2006), 12343-12353.

[8] T. H. Lee, Electrical solitons come of age, Nature, 440 (2006), 36-37.

[9] H. Nagashima and Y. Amagishi, Experiment on the Toda lattice using nonlinear transmission lines, J. Phys. Soc. Jpn., 45 (1978), 680-688.

[10] A. Nakamura, Interaction of Toda lattice soliton with an impurity atom, Prog. Theor. Phys., 59 (1978), 1447-1460.

[11] A. Nakamura and S. Takeno, Scattering of a soliton by an impurity atom in the Toda lattice and localized modes, Prog. Thoer. Phys., 58 (1977), 1074-1076. 
[12] K. Steiglitz, Time-gated Manakov spatial solitons are computationally universal, Phys. Rev. E, 63 (2000), 016608.

[13] H. Tian, J. Tian, and Y. Ji, Bright and dark solitons in quadratic nonlinear periodic structures and application to an all-optical logic gate, J. Phys. B: At. Mol. Opt., 40 (2007), 1391-1402.

[14] M. Toda, Vibration of a chain with nonlinear interaction, J. Phys. Soc. Jpn., 22 (1967), 431-436.

[15] L. Vergara and B. A. Malomed, Suppression of the generation of defect modes by a moving soliton in an inhomogeneous Toda lattice, Phys. Rev. E, 77 (2008), 047601.

[16] S. Watanabe and M. Toda, Experiment on soliton-impurity interaction in nonlinear lattice using LC circuit, J. Phys. Soc. Jpn., 50 (1981), 34433450 .

[17] N. Yajima, Scattering of lattice solitons from a mass impurity, Phys. Scr., 20 (1979), 431-434.

Received: February, 2012 